\title{
Valor diagnóstico de la Escala de Ansiedad y Depresión de Goldberg (EAD-G) en adultos cubanos*
}

\author{
Diagnostic usefulness of Anxiety and Depression \\ Scale Goldberg (EAD-G) in Cuban adults
}

Recibido: 05 de junio de 2013 | Aceptado: 25 de septiembre de 2015

\author{
Marta Martín CARbONEll ** \\ Universidad Cooperativa de Colombia, Santa Marta, \\ Colombia \\ RAQuel PÉRez DÍAZ **** \\ Centro de Investigaciones sobre Longevidad, \\ Envejecimiento y Salud, La Habana, Cuba \\ ANTONIO RIQUELME MARÍN **** \\ Universidad de Murcia, España
}

doi:10.11144/Javeriana.upsy15-1.vdea

Martín Carbonell, M. (2016). Valor diagnóstico de la Escala de Ansiedad y Depresión de Goldberg (EAD-G) en adultos cubanos. Universitas Psychologica, 15(1) 177-192. http://dx.doi.org/10.11144/ Javeriana.upsy15-1.vdea

* Artículo de investigación. Proyecto financiado por la Fundación Séneca, Ayudas a la consolidación de grupos de investigación (Grupos precompetitivos) 2009, 11946/PPC/09

** Docente-Investigadora. Facultad de Psicología. Correo electrónico: martamartincarbonell@hotmail.com

**** Departamento de Psicología Centro de Investigaciones sobre Longevidad, Envejecimiento y Salud.

****** Departamento de Personalidad, Evaluación y Tratamientos Psicológicos Facultad de Psicología.

\section{RESUMEN}

El estudio tiene como objetivo obtener información para población cubana acerca de la validez predictiva de la EADG para detectar personas con trastornos psicopatológicos, así como para diferenciar ansiedad y depresión. Se trabajó con una muestra no probabilística, integrada por 548 sujetos, de los cuales el 31,2\% tenían un trastorno psicopatológico diagnosticado por un psiquiatra o psicólogo. Para el análisis de los datos se utilizó la metodología de análisis de las curvas ROC. Se encontró que la EADG mostró un valor predictivo adecuado para identificar a personas con trastornos psicopatológicos, con escasa capacidad para distinguir trastornos de ansiedad y depresión. Estos resultados apoyan la estrategia evaluativa recomendada por los autores de aplicar primeramente los ítems de despistaje.

Palabras clave

Escala de Ansiedad y Depresión de Goldberg; EADG; evaluación psicológica; psicometría

\section{A B S T R A C T}

The study has as objective to obtain information for Cuban population about the predictive validity of the EADG for detecting people with psychopathologic dysfunctions, as well as to differentiate anxiety and depression. It worked with a non probabilistic sample, integrated by 548 subjects, of which $31.2 \%$ had a psychopathologic disease diagnosed by a psychiatrist or psychologist. For the analysis of the data, the methodology analysis of the curves ROC was used. It was found that the EADG showed an appropriate predictive value to identify people with psychopathologic dysfunctions, and difficulties to distinguish dysfunctions of anxiety and depression. Results support the evaluative strategy recommended by the authors of applying the screening items firstly.

Keywords

Anxiety and Depression Scale; GADS; psychological evaluation; psychometry 


\section{Introducción}

La Declaración de Consenso emitida por el Grupo de Consenso Internacional sobre Depresión y Ansiedad (Ballenger et al., 2001) subraya la prevalencia de estos trastornos en todas las naciones, y que existen diferencias culturales en la presentación de los síntomas. Estas diferencias se explican en primer lugar, porque "la propia psiquiatría es una institución cultural" (Kirmayer, 2001, p. 22) lo que implica que la evaluación acerca de qué, o por qué, un comportamiento determinado se puede considerar como un síntoma, depende del bagaje etnocultural de los profesionales. Por ejemplo, los límites sobre lo "normal" y lo "patológico" en la expresión y la regulación emocional están culturalmente determinados: una muestra de ello es que en Estados Unidos la depresión se asocia a connotaciones de pérdida de fortaleza personal, lo que contribuye a que sea estigmatizada, por lo que ciertos grupos tienden a minimizar o negar los componentes afectivos del distrés y le dan prioridad a las quejas somáticas, que son socialmente más aceptables (Kirmayer, 2001)

La Guía Latinoamericana de Diagnóstico Psiquiátrico - GLADP- (APAL, 2003) surge como respuesta a la necesidad de la psiquiatría latinoamericana de adaptar la clasificación internacional de los trastornos psiquiátricos de la Organización Mundial de la Salud (Capítulo de Trastornos Mentales de la $10^{\mathrm{ma}}$ Clasificación Internacional de las Enfermedades-CIE-10) y de los manuales diagnósticos norteamericanos (como el DSM) a las necesidades particulares de la población de América Latina. Se reconoce por ejemplo la presencia de síndromes locales de importancia en nuestro medio, tales como el "susto" o el "ataque de nervios", que obtienen solo una cobertura marginal en otros sistemas clasificatorios.

Como se plantea en la GLADP:

Las necesidades de América Latina en materia de nosología psiquiátrica son particulares ya sólo por el hecho innegable de que la manera de enfermar de su población está influida fuertemente por su cultura. También, las circunstancias socioeconómicas de amplios sectores de esta población obligan a enfati- zar aspectos de prevención y promoción de la salud, que requieren un enfoque particular a la manera de organizar los trastornos que aquejan a estos pacientes. (APAL, 2003, p. 9)

En Cuba no se ha realizado un estudio nacional que describa la prevalencia ni las particularidades de presentación de los trastornos de ansiedad y depresión, pero existen reportes de investigaciones realizadas sobre la prevalencia de la depresión en regiones específicas que estiman cifras que van del 12 al 18\% (Fors, 2011). En una revisión sistemática sobre estudios de prevalencia de problemas de salud mental que incluyó investigaciones comunitarias realizadas en seis países de América Latina se encontró que los trastornos que afectaron al mayor número de adultos fueron la depresión mayor, la distimia, los trastornos de ansiedad generalizada, y el abuso o la dependencia del alcohol. Sin embargo, según concluyeron los autores, posiblemente se haya subestimado la magnitud del problema pues también hallaron que más de la mitad de las personas afectadas por trastornos de ansiedad no habían recibido tratamiento psiquiátrico (Kohn et al., 2005)

Lamentablemente, es muy probable que la demanda real de atención especializada para los problemas de ansiedad y depresión sea incluso superior a la percibida por las dificultades que tienen los médicos para detectar los trastornos psiquiátricos (Cepoiu et al., 2008; Mitchell, Vaze, \& Rao, 2009; Mc Gorry \& Van Os, 2013) entre las que se mencionan la sobrecarga asistencial y la falta de tiempo, así como la poca familiaridad con los diagnósticos psicopatológicos y el hecho de que la mayoría de los pacientes con trastornos mentales que acuden al médico de atención primaria consultan por síntomas "físicos" (insomnio, cefalea, fatiga, pérdida de peso, etc.) que pueden ser atribuidos a otras enfermedades (Latorre et al., 2012).

Aunque la entrevista clínica constituye sin duda el mejor método para el descubrimiento de los trastornos psicopatológicos, requiere una serie de habilidades por parte del entrevistador que no siempre están presentes, por lo que se plantea que la integración de algunos cuestionarios en el seno de la entrevista puede facilitar y mejorar la detección de 
psicopatología (Fernández et al., 2007). Un estudio meta-analítico afirma que los instrumentos cortos son útiles para diagnosticar la depresión, siempre y cuando sea posible realizar un segundo análisis de los casos identificados (Mitchell, Vaze, \& Rao, 2009).

Se han recomendado diversos instrumentos como el cuestionario PRIME - Primary Care Evaluation of Mental Disorders- (Baca et al., 1999), la Escala de Ansiedad y Depresión Hospitalaria- HASD-(A. Bunevicius, Peceliuniene, Mickuviene, Valius, \& R. Bunevicius, 2007), o el PHQ-9 _Patient Health Questionnaire - (Cameron, Crawford, \& Lawton, 2008); pero ninguno de ellos se ha validado ni están disponibles para su aplicación en población cubana. Sin embargo en Cuba hay experiencias con la Escala de Ansiedad y Depresión de Goldberg (EADG), que ha sido considerada por diversos autores entre los más prometedores de los numerosos instrumentos desarrollado para el pesquisaje (Latorre et al., 2012; Gilbody, House, \& Sheldon, 2009).

La EADG (Goldberg Anxiety and Depression Scale -GADS- en inglés) fue propuesta por su autor en 1988 a partir de una versión modificada de la Psychiatric Assessment Schedule, con la finalidad de lograr una entrevista de corta duración que pudiera ser utilizada por médicos no psiquiatras como instrumento de pesquisaje (Goldberg, Bridges, Duncan-Jones, \& Grayson, 1988). Su sencillez, unida a su capacidad de aportar información dimensional sobre la gravedad, han hecho que esta escala haya sido ampliamente recomendada como instrumento de cribaje, tanto con fines asistenciales como epidemiológicos, y/o como guía de la entrevista clínica en el ámbito de la atención primaria (López, Gabarrón, \& Ruiz, 2011)

Ha sido adaptada en numerosos países, la versión en castellano ha sido validada por Montón, Echevarría, y Campos (1993) en población española. Según estos autores, tiene una sensibilidad (83.1\%), especificidad (81.8\%) y valor predictivo positivo (95.3\%) adecuados. La dimensionalidad de la EADG se ha investigado mediante el análisis de rasgos latentes con variables dicotómicas (Goldberg et al.,1988; Mackinnon, Christersen, \& Form, 1994) y también en el estudio longitudinal australiano de salud de la mujer (ALSWH, 2011). También se ha investigado mediante el análisis de componentes principales en su validación italiana (Magnavita, 2007). En todos estos estudios, se ha encontrado un factor que agrupa los 18 ítems para explicar el grueso de la varianza, así como factores específicos para los ítems de ansiedad y depresión.

La validez convergente de la EADG con otros instrumentos de cribaje de amplia difusión como el Cuestionario de Salud de Goldberg (GHQ-28), el MINI (Mini International Neuropsychiatic Interview) o el PRIME-MD (Primary Care Evaluation of Mental Disorders) es alta (Martínez Bernardos, Baylín-Larios, \& Ortiz-Jiménez, 1999) con valores superiores al 70\% (Tizón et al., 1999).

El presente estudio tiene como objetivo obtener información para población cubana acerca de la validez predictiva de la EADG para detectar personas con trastornos psicopatológicos, así como para diferenciar ansiedad y depresión.

\section{Método}

\section{Participantes}

Se trabajó con una muestra no probabilística, integrada por 548 sujetos, de los cuales el $62.7 \%$ eran mujeres. Todos los casos eran adultos con edades entre 22 y 103 años, el promedio de edad de la muestra fue de 58.44, con una desviación típica $=17.14$. El 51.5\% carecía de un vínculo laboral estable, pues el 32.1\% eran personas jubiladas, el 17.5\% eran mujeres que siempre se habían dedicado exclusivamente a las tareas del hogar, el 1.7\% eran estudiantes y el resto eran personas en paro. Entre los que tenían vínculo laboral se encontraba un $35.4 \%$ de profesionales de nivel superior, un $45.6 \%$ de técnicos o administrativos y el resto trabajaban en diversos oficios. Predominaba la escolaridad media y superior $(0.4 \%$ no sabían leer ni escribir, $16.1 \%$ tenían estudios primarios, $20.1 \%$ estudios secundarios, $35.2 \%$ nivel de preuniversitario y el resto eran graduados universitarios). El $49.6 \%$ tenía pareja estable.

170 sujetos ( $31.2 \%$ de los casos) tenían un trastorno psicopatológico diagnosticado por un psiquiatra o psicólogo y recibían atención especializada en alguna de las instituciones de salud de la capital (ver procedimiento). 


\section{Instrumento}

Escala de Ansiedad y Depresión de Goldberg -EADG (Goldberg et al., 1988): Como indica su nombre, consta de dos sub-escalas, una de ansiedad y otra de depresión. Cada sub-escala tiene 9 ítems de respuesta dicotómica (Si/No) y se estructuran en 4 ítems iniciales de despistaje, para determinar si es o no probable que exista un trastorno mental, y un segundo grupo de 5 ítems que se formulan si se obtiene respuestas positivas a las preguntas de despistaje ( 2 o más para ansiedad y más de 1 para depresión), pero en la investigación se suele aplicar la escala completa, y de esa manera fue utilizada en el presente estudio.

Se da una puntuación independiente para cada escala, con un punto para cada respuesta afirmativa. Goldberg et al. (1988) han propuesto como puntos de corte $\geq 4$ para la escala de ansiedad, y $\geq 2$ para la de depresión. Para estos valores se ha reportado una sensibilidad del $83 \%$ y una especificidad del $82 \%$. Se plantea que existe una mejora de la sensibilidad al aumentar la gravedad del trastorno psicopatológico, obteniéndose puntuaciones más altas que pueden proporcionar una medida dimensional de la gravedad de cada trastorno por separado (López et al., 2011).

\section{Procedimiento}

Esta investigación forma parte de un estudio más amplio dedicado a indagar en las relaciones entre la afectividad y la depresión en personas de Cuba y España ${ }^{1}$. Además de la EADG, en la mencionada investigación se utilizó el Inventario de Afecto positivo y Afecto negativo (PANAS) (Watson, Clark, \& Tellegen, 1988) en la versión española llevada a cabo por Sandín et al. (1999), y una entrevista estructurada con preguntas referidas a variables sociodemográficas y de salud. Para el presente estudio se utilizaron los datos de una parte de la muestra cubana, que se terminaron de recolectar en diciembre del 2011.

1 Proyecto financiado por la Fundación Séneca, Ayudas a la consolidación de grupos de investigación (Grupos precompetitivos) 2009, 11946/PPC/09
Los participantes "sin trastornos psicopatológicos" se reclutaron en la ciudad de la Habana, en barrios de los municipios Cerro y Marianao, también en un hogar de día y en una residencia de ancianos. Para conformar la muestra se utilizó la técnica "bola de nieve", consistente en la ampliación progresiva de aquella a partir de los contactos proporcionados por los participantes inicialmente seleccionados por los miembros del equipo de investigación siguiendo un criterio de conveniencia. Se excluyeron las personas que referían tener malestar emocional intenso y/o estar en tratamiento con psicólogo o psiquiatra. Dos estudiantes de último año de Psicología especialmente entrenados, aplicaron los instrumentos para la recogida de información.

Las personas con trastornos psicopatológicos fueron contactadas gracias a la colaboración del psiquiatra o psicólogo responsable de su atención en las consultas externas de servicios de psiquiatría de diversos hospitales de la Habana y en el Centro de Investigaciones sobre Longevidad, Envejecimiento y Salud (CITED). El diagnóstico fue realizado por el profesional responsable de la atención del paciente, previamente a la aplicación de la EAD-G; y fue copiado textualmente de la Historia Clínica oficial de la institución de salud. Al igual que para la muestra no clínica, los instrumentos para la recogida de información fueron aplicados por dos estudiantes de último año de Psicología especialmente entrenados.

A todos los participantes se les explicó el objetivo de la investigación y se les solicitó de manera oral su consentimiento para utilizar la información proporcionada con fines de investigación, asegurando la privacidad y confidencialidad. Así mismo, se les aseguró la posibilidad de abandonar el estudio en el momento que lo desearan, sin necesidad de dar explicaciones y sin que esto les acarreara algún tipo de consecuencia. Se solicitó autorización a las personas pertinentes para la aplicación de las pruebas en cada uno de los centros.

\section{Análisis de los datos}

El almacenamiento y procesamiento de los datos se hizo mediante el paquete estadístico SPSS 
versión 20. Para estudiar la validez predictiva de la EAG para diferenciar los trastornos de ansiedad y depresión, así como para identificar a personas con trastornos psicopatológicos, se utilizó la metodología análisis de las curvas ROC (Franco \& Vivo, 2007).

\section{Resultados}

Clasificación de las personas con trastornos psicopatológicos según el diagnóstico realizado por el profesional responsable de su atención

La Tabla 1 informa la frecuencia de casos de acuerdo al diagnóstico emitido por el profesional.
Como puede observarse, aparecen algunos diagnósticos que no están reconocidos en ninguno de los manuales de clasificación como por ejemplo, "sintomatología relevante de ansiedad y depresión" o "reacción de duelo prolongado".

Capacidad de la EADG para distinguir a las personas con trastornos psicopatológicos

En la Tabla 2 y el Gráfico 1 se muestra que la EAD. $\mathrm{G}$ total, así como todas sus sub-escalas, tienen valor discriminante estadísticamente significativo pues son capaces de identificar a los personas con diagnósticos de trastornos psicopatológicos mejor que un clasificador aleatorio $(\mathrm{p}<0.05)$.

\section{TABLA 1}

Distribución de los casos por grupos de estudio, según diagnóstico emitido por el profesional

\begin{tabular}{llc}
\hline Grupo de estudio & DIAGNÓSTICO & Frecuencia \\
\hline DEPRESIÓN & Episodio depresivo mayor & 37 \\
& Trastorno depresivo no especificado & 11 \\
& Sintomatología depresiva relevante & 2 \\
& Reacción de duelo prolongado & 1 \\
& Distimia & 19 \\
& TOTAL & 70 \\
\hline ANSIEDAD & Trastorno ansiedad generalizada & 28 \\
& trastorno de pánico con o sin agorafobia & 11 \\
& Fobia & 21 \\
& Trastorno de ansiedad sin especificar & 5 \\
& trastorno adaptativo ansioso & 3 \\
& trastorno adaptativo depresivo & 3 \\
& trastorno obsesivo compulsivo & 3 \\
& trastorno de la personalidad & 5 \\
& Trastorno Hipocondriaco & 1 \\
& TOTAL & 80 \\
\hline MIXTOS & Reacción mixta de ansiedad y depresión & 4 \\
& Sintomatología relevante de ansiedad y depresión & 8 \\
& TOTAL & 12 \\
\hline OTROS DIAGNÓSTICOS & Neurosis histérica & 2 \\
& Neurosis de ansiedad & 4 \\
\hline TOTAL & Depresión con intento suicida & 1 \\
& Neurastenia & 2 \\
& TOTAL & 8 \\
\hline
\end{tabular}

Fuente: elaboración propia 
TABLA 2

Área bajo la curva de la EADG

\begin{tabular}{|c|c|c|c|c|c|}
\hline \multirow{2}{*}{ Variables resultado de contraste } & \multirow{2}{*}{ Área } & \multirow{2}{*}{ Error típ. ${ }^{a}$} & \multirow{2}{*}{ Sig. asintóticab } & \multicolumn{2}{|c|}{$\begin{array}{l}\text { Intervalo de confianza } \\
\text { asintótico al } 95 \%\end{array}$} \\
\hline & & & & $\begin{array}{l}\text { Límite } \\
\text { inferior }\end{array}$ & $\begin{array}{l}\text { Límite } \\
\text { superior }\end{array}$ \\
\hline \multicolumn{6}{|l|}{ Ítems recomendados para el despistaje } \\
\hline Ítems de despistaje de la sub escala ansiedad & 0.635 & 0.024 & 0 & 0.587 & 0.683 \\
\hline Ítems de despistaje de la sub-escala de depresión & 0.707 & 0.023 & 0 & 0.661 & 0.753 \\
\hline \multicolumn{6}{|l|}{ Sub-escalas } \\
\hline EADG ANSIEDAD & 0.646 & 0.024 & 0 & 0.599 & 0.694 \\
\hline EADG DEPRESION & 0.688 & 0.024 & 0 & 0.640 & 0.735 \\
\hline \multicolumn{6}{|l|}{ Escala completa } \\
\hline EADG Total & 0.696 & 0.022 & 0 & 0.653 & 0.740 \\
\hline
\end{tabular}

Fuente: elaboración propia

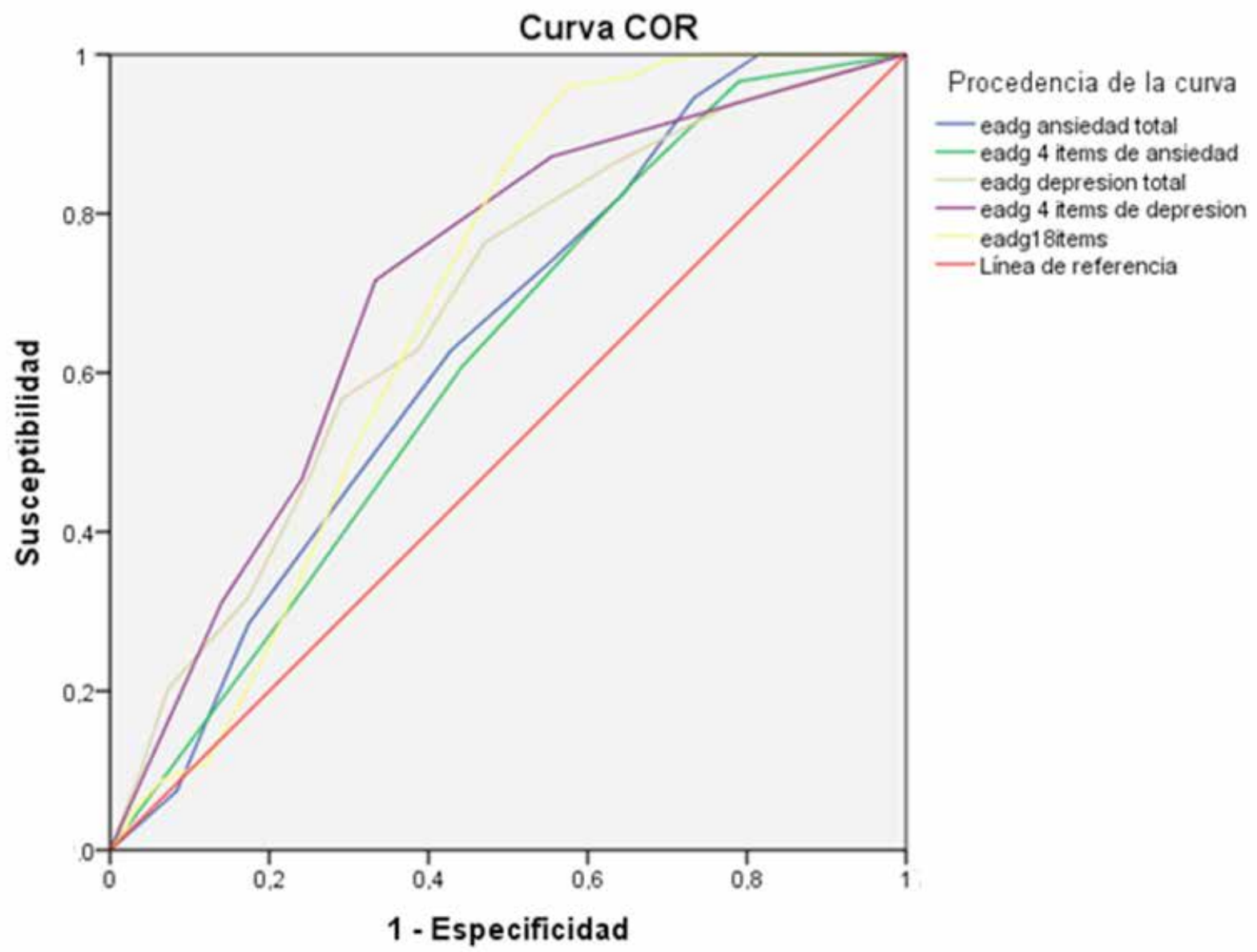

Los segmentos diagonales son producidos por los empates.

Gráfico 1. Curvas ROC de la EAD-G y sus sub-escalas. Muestra para cada posible punto de corte, la medida de rendimiento teniendo en cuenta su sensibilidad y especificidad. Intuitivamente, se puede afirmar que los puntos más alejados de la diagonal tienen mayor poder de clasificación 
En la Tabla 3 se ofrecen los valores predictivos específicos de las sub-escalas de ansiedad y depresión para distinguir a las personas que tenían algún trastorno psicopatológico.

\section{Capacidad de la EADG para diferenciar ansiedad y depresión}

Se utilizó nuevamente la metodología de la curvas ROC, pero para un sub-grupo de personas que tenían un diagnóstico clínico de ansiedad (67 casos) y depresión (73 casos).

\section{Capacidad de la EADG para identificar a personas con depresión.}

Como se observa en la Tabla 4 y en el Gráfico 2, la sub-escala de depresión clasifica muy bien los casos diagnosticados como deprimidos, pues es capaz de contar como deprimidos al $87 \%$ de las personas que han tenido este diagnóstico, no así la sub-escala de ansiedad, que clasifica sólo al 21\% de los pacientes diagnosticados como tal, aunque las dos son capaces de discriminar significativamente $(\mathrm{p}<0.05)$.

La Tabla 5 ofrece información sobre los valores predictivos de las distintas puntuaciones de la EAD$\mathrm{G}$ para identificar a las personas con depresión.

\section{Capacidad de la EADG para identificar a personas con ansiedad.}

Los resultados se muestran en las tablas 6 y 7 y en el Gráfico 3. En este caso, la sub-escala de ansiedad muestra buena capacidad para identificar los casos que han sido diagnosticados como tales (79\%), no así la sub-escala de depresión qué solo detecta al $12 \%$ de los ansiosos, aunque ambas resultan clasificadores mejores que el azar $(\mathrm{p}<0.05)$.

TABLA 3

Coordenadas de la curva para casos con y sin psicopatología de los 4 items de despistaje de las sub-escalas de ansiedad y depresión de la EADG

\begin{tabular}{l|c|c|c}
\hline Variables resultado de contraste & Positivo si es mayor o igual que $^{\mathrm{a}}$ & Sensibilidad & 1 - Especificidad \\
\hline \multirow{4}{*}{ Subescala Ansiedad } & -1 & 1 & 1 \\
& 0.5 & 0.970 & 0.790 \\
& 1.5 & 0.857 & 0.653 \\
& 2.5 & 0.631 & 0.440 \\
& 3.5 & 0.357 & 0.232 \\
& 5 & 0 & 0 \\
\hline \multirow{3}{*}{ Subescala Depresión } & -1 & 1 & 1 \\
& 0.5 & 0.875 & 0.560 \\
& 1.5 & 0.714 & 0.336 \\
& 2.5 & 0.458 & 0.238 \\
& 3.5 & 0.304 & 0.139 \\
\hline
\end{tabular}

Fuente: elaboración propia

TABLA 4

Área bajo la curva de las sub-escalas de la EDAG para discriminar deprimidos de ansiosos

\begin{tabular}{cccccc}
\hline Variables resultado de contraste & Área & $\begin{array}{c}\text { Error típ.a } \\
\text { Límite inferior }\end{array}$ & $\begin{array}{c}\text { Sig. asintóticab } \\
\text { Límite superior }\end{array}$ & \multicolumn{2}{c}{ Intervalo de confianza asintótico al } \\
$95 \%$
\end{tabular}

Fuente: elaboración propia 


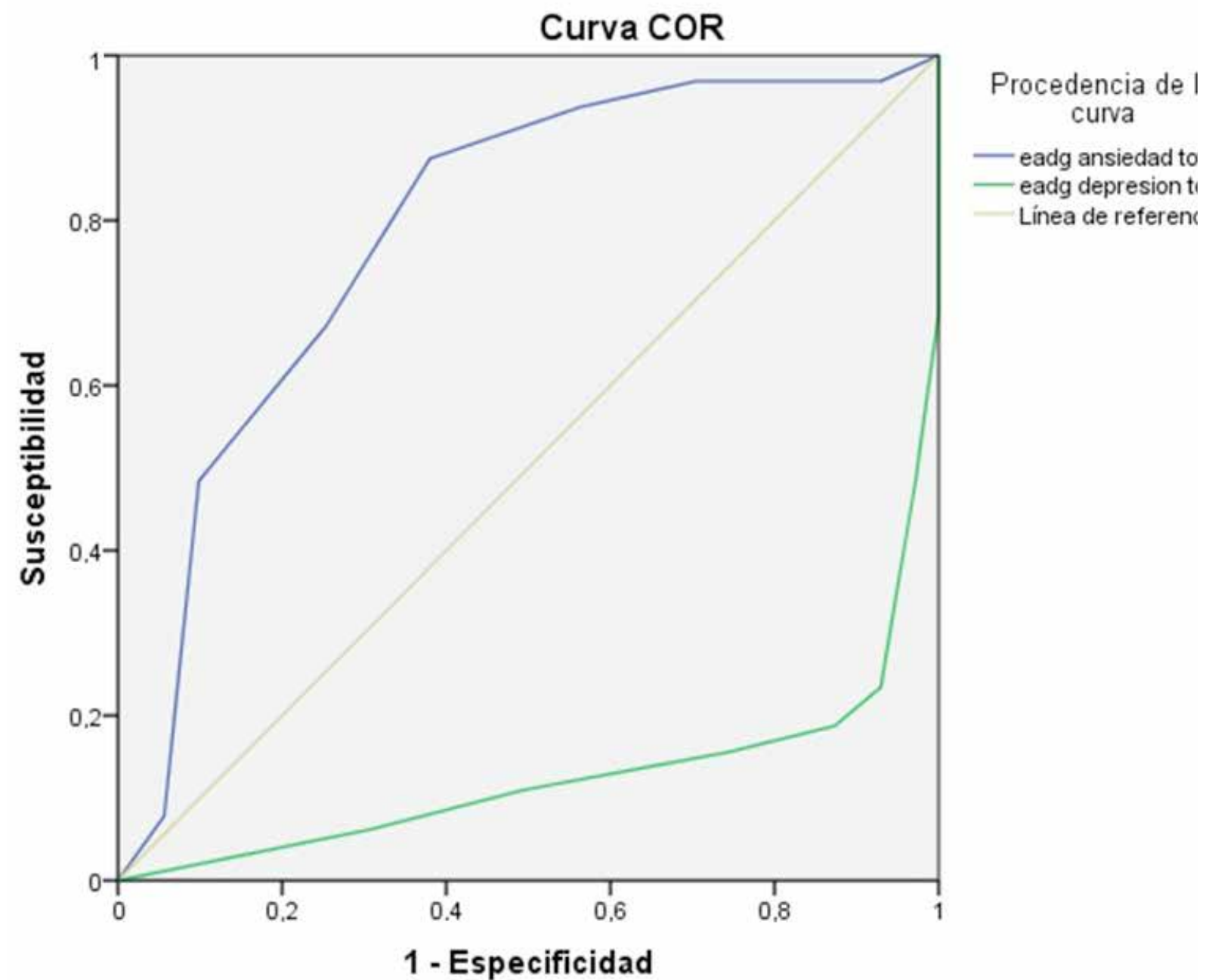

Los segmentos diagonales son producidos por los empates.

Gráfico 2. Curva ROC de las subescalas de la EADG para depresión

Fuente: elaboración propia

\section{Discusión}

Antes de entrar a valorar los resultados obtenidos con la EADG, conviene comentar la información que se muestra en la Tabla 1 sobre los diagnósticos recibidos por los pacientes. Como se observa en la misma, no existe un criterio uniforme y aparecen diagnósticos provenientes de diferentes manuales (GLADP, CIE-10 y DSM-IV). Aunque en Cuba se recomienda usar la GLADP para los diagnósticos psiquiátricos, hay variabilidad pues algunos profesionales usan el DSM-IV (vigente en el momento en que se recogieron los datos) o la CIE-10, en dependencia de sus preferencias. Por otra parte, también aparecen diagnósticos que no están reconocidos en ningún manual de clasificación, lo que ocurre frecuentemente en la práctica clínica (Fernández et al., 2010), quizás porque los médicos y psicólogos en la práctica usan más una perspectiva dimensional o etiológica, que categorial (Cano, Martín, Wood, Dongil \& Latorre, 2012).

Otra explicación posible es que existe una discordancia importante y reconocida entre lo que preocupa a los investigadores y lo que realmente 
TABLA 5

Coordenadas de la curva de la EADG para deprimidos

\begin{tabular}{cccc}
\hline & Coordenadas de la curva & & \\
\hline Variables resultado de contraste & Positivo si es mayor o igual que & & \\
\hline & 1 & Sensibilidad & 1 - Especificidad \\
\hline \multirow{2}{*}{ EADG ANSIEDAD } & 2.5 & 1 & 1 \\
& 3.5 & 0.930 & 0.969 \\
& 4.5 & 0.704 & 0.969 \\
& 5.5 & 0.563 & 0.938 \\
& 6.5 & 0.380 & 0.875 \\
& 7.5 & 0.254 & 0.672 \\
EADG DEPRESION & 8.5 & 0.099 & 0.484 \\
& 10 & 0.056 & 0.078 \\
& -1 & 0 & 0 \\
\hline 0.5 & 1 & 1 \\
& 1.5 & 1 & 0.859 \\
& 2.5 & 1 & 0.688 \\
& 3.5 & 0.972 & 0.484 \\
& 4.5 & 0.930 & 0.234 \\
& 5.5 & 0.873 & 0.188 \\
& 6.5 & 0.746 & 0.156 \\
& 7.5 & 0.493 & 0.109 \\
& 8.5 & 0.310 & 0.063 \\
& 10 & 0.155 & 0.031 \\
\hline
\end{tabular}

Fuente: elaboración propia

TABLA 6

Área bajo la curva

\begin{tabular}{lccccc}
\hline Variables resultado de contraste & Área & Error típ. $^{\text {a }}$ & Sig. asintótica & \multicolumn{2}{c}{ Intervalo de confianza asintótico al 95\% } \\
\cline { 5 - 6 } & & & & Límite inferior & Límite superior \\
\hline EADG ANSIEDAD & 0.790 & 0.040 & 0 & 0.711 & 0.868 \\
\hline EADG DEPRESION & 0.124 & 0.033 & 0 & 0.061 & 0.188 \\
\hline
\end{tabular}

Fuente: elaboración propia

ocupa a los clínicos en su práctica habitual. En el año 2013, un grupo de profesionales e instituciones de diversos países publicaron el "Manifiesto a favor de una psicopatología clínica no estadística" que plantea que todo conocimiento clínico debe ser considerado como una aproximación teórica y de ninguna manera puede ser asumido como "la verdad", y sobre todo se alerta contra la códigos de diagnóstico y tratamiento únicos, obligatorios y universales, concebidos desde estructuras de poder económico y científico. También la sección de psi- cología clínica de la Asociación Británica de Psicología (División de Psicología Clínica -DCP-, 2013) se opone a la aplicación del modelo biomédico para la comprensión de los trastornos mentales y llama a realizar un cambio de paradigma hacia un sistema conceptual que no esté basado en un modelo de enfermedad sino en un modelo bio-psico-social, multifactorial, que tenga en cuenta los contextos en que aparecen la ansiedad y las alteraciones de la conducta y que reconozca la complejidad de las interacciones. 
TABLA 7

Coordenadas de la curva

\begin{tabular}{cccc}
\hline Variables resultado de contraste & Positivo si es mayor o igual quea & Sensibilidad & 1 - Especificidad \\
\hline & 1 & 1 & 1 \\
& 2.5 & 0.969 & 0.930 \\
EADG ANSIEDAD & 3.5 & 0.969 & 0.704 \\
& 4.5 & 0.938 & 0.563 \\
& 5.5 & 0.875 & 0.380 \\
& 6.5 & 0.672 & 0.254 \\
& 7.5 & 0.484 & 0.099 \\
& 8.5 & 0.078 & 0.056 \\
& 10 & 0 & 0 \\
\hline & -1 & 1 & 1 \\
& 0.5 & 0.859 & 1 \\
& 1.5 & 0.688 & 1 \\
& 2.5 & 0.484 & 0.972 \\
& 3.5 & 0.234 & 0.930 \\
& 4.5 & 0.188 & 0.873 \\
& 5.5 & 0.156 & 0.746 \\
& 6.5 & 0.109 & 0.493 \\
& 7.5 & 0.063 & 0.310 \\
& 8.5 & 0.031 & 0.155 \\
\hline
\end{tabular}

Fuente: elaboración propia

A los efectos de este estudio, se consideró que dado que el objetivo era explorar la capacidad diagnóstica de la EADG, se podía admitir cierta imprecisión en las categorías diagnósticas pues el interés está centrado en la presencia de sintomatología ansiosa y/o depresiva, que es frecuente en muchos y disímiles trastornos.

En este sentido, la EAD-G muestra tener un adecuado valor predictivo para identificar a personas con trastornos psicopatológicos, teniendo en cuenta que se utilizó como "patrón de oro" el diagnóstico realizado por especialistas en salud mental quienes estuvieron "a ciegas" de los resultados de su aplicación.

Para los puntos de corte recomendados por Goldberg et al. (1988) se obtiene una adecuada sensibilidad y especificidad pues como puede observarse en la Tabla 3, una puntuación mayor a 1.5 en la sub-escala de depresión tiene una sensibilidad de $71 \%$ y una especificidad de $77 \%$ (1-especifici$\mathrm{dad}=0.336)$, o sea, clasifica correctamente al $71 \%$ de los casos con trastornos psicopatológicos y al $77 \%$ de las personas sin trastornos. En el caso de la sub-escala de ansiedad, también se obtiene para esta puntuación una alta sensibilidad (85\%) aunque menor especificidad (65\%). Así, para la identificación de las personas con trastornos psicopatológicos, se puede considerar que todas las sub-escalas tienen un rendimiento "justo" de acuerdo a Franco y Vivo (2007) pues los valores del área bajo la curva se encuentran en todos los casos entre 0.50 y 0.75 . Esto apoya que sea usada con fines de cribaje, pero por supuesto, no puede sustituir al diagnóstico clínico.

En el presente estudio no se investigó si existían diferencias estadísticamente significativas en cuanto a la capacidad discriminante de las diferentes sub-escalas, pero si tomamos en cuenta las recomendaciones de Franco y Vivo (2007) para estimar la exactitud de un clasificador, observamos que los 4 ítems recomendados para el despistaje identifican tan bien a las personas con trastornos psicopatológicos como las sub-escalas completas de 


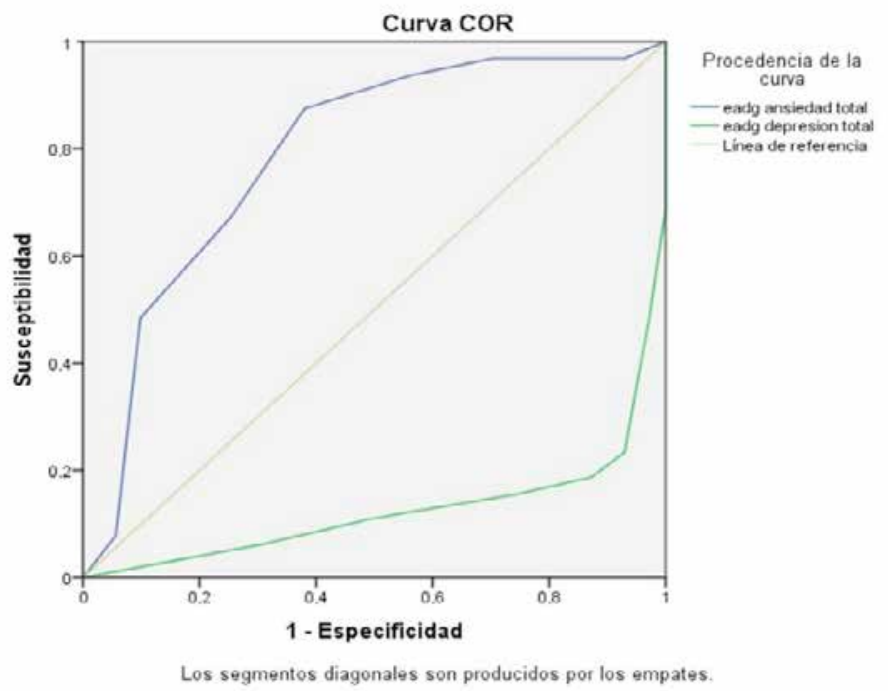

Gráfico 3. Curva ROC de las subescalas de la EADG para ansiedad

Fuente: elaboración propia

9 ítems. Igualmente, parece que aplicar los 18 ítems de la prueba completa aporta poco a la exclusión de psicopatología. Por lo tanto, parece recomendable usar sólo los ítems de despistaje en el contexto de la entrevista médica habitual, tal y como han recomendado diversos autores (Arrol, Khin, \& Kerse, 2003; A. Bunevicius et al., 2007 y Latorre et al., 2012, entre otros) ya que emplear pocas preguntas con un alto poder discriminante permite que se incremente y mejore la identificación de personas con trastornos psicopatológicos en el nivel primario de atención, como reclaman las autoridades sanitarias (Ballenger et al., 2001; Botto, Acuña, \& Jiménez, 2014).

Se debe recordar que Goldberg et al. (1988) crearon esta prueba con el propósito de optimizar lo más posible el procedimiento para detectar casos con psicopatología, teniendo en cuenta la falta de experiencia en psiquiatría de los médicos generalistas y del nivel primario, así como la gran cantidad de pacientes que deben atender usualmente. Para ello utilizaron estrategias de análisis derivadas de la Teoría de Respuesta al Ítem (Muñiz, 1997; Rasch, 1960), que parte de tener en cuenta que no todos los ítems tienen igual capacidad diagnóstica, pues las respuestas se determinan por una parte, por el grado en que el problema está presente en el paciente, y por otra parte, por el grado de dificultad para ser respondido que tenga el propio ítem.

Así, seleccionaron las preguntas que habían mostrado mayor capacidad discriminante de ansiedad y depresión respectivamente en un estudio previo (Goldberg, Bridges, Duncan-Jones, \& Grayson, 1987); y otro grupo de preguntas que solo se formulan si las anteriores son positivas, y van más orientadas a investigar la severidad del trastorno. Nuestros resultados apoyan la estrategia evaluativa recomendada por los autores de aplicar primeramente los ítems de despistaje, y solo continuar con los restantes si hay más de dos respuestas positivas en la sub-escala de ansiedad y más de una en la sub-escala de depresión. Esto deberá corroborarse en trabajos posteriores, pues en el presente se aplicó la escala completa de una vez y conjuntamente con otros instrumentos.

Los puntos de corte que recomiendan Goldberg et al. (1988) en la muestra del presente estudio aparecen con mejor sensibilidad que especificidad, al igual que reportan otros autores (Lobo et al., 1993), por lo que deberá tenerse en cuenta que probablemente la EADG no sea la herramienta indicada para aquellos estudios en los que interese privilegiar la identificación de "verdaderos positivos". Sin embargo el costo de los "falsos negativos" en términos 
socioeconómicos para los sistemas sanitarios, y de sufrimiento para las personas que padecen de ansiedad y depresión sin recibir tratamiento, justifica que se incorpore a la entrevista médica habitual los ítems de despistaje (van Beljouw, Verhaak, Cuipjpers, van Marwijk \& Pennix, 2010; Cano, Martín, Wood, Dongil, \& Latorre, 2012).

Otro de los fines de esta investigación fue estudiar la capacidad de la EADG para la diferenciación de la ansiedad y la depresión.

El examen visual de los gráficos 2 y 3 ilustra que las sub-escalas de depresión y de ansiedad son clasificadores "estrictos" del trastorno respectivo, pues detectan muy bien los verdaderos positivos (87\% la sub-escala de depresión y $97.2 \%$ la subescala de ansiedad), pero también muestra que la escala de ansiedad puede ser evaluada como un clasificador "tolerante" para los casos con diagnóstico de depresión, y viceversa, la sub-escala de depresión es también un clasificador "tolerante" de los casos diagnosticados como ansiosos, pues ambas ocupan el espacio ROC por debajo de la diagonal, lo que señala que ellas identifican significativamente mayor cantidad de falsos positivos que un clasificador aleatorio, pues admiten como positivos a casos que en realidad son negativos (Franco \& Vivo, 2007).

Este resultado podría aportar hasta cierto punto, evidencias de la poca validez discriminante de estas sub-escalas (Martínez-García \& MartínezCaro, 2009); y lo que es más importante, acerca de la validez teórica y clínica de la diferenciación entre ansiedad y depresión, sobre la cual aún no se ha llegado a consenso (García, Saldívar, LLanes, \& Sánchez, 2011; Gruszczyńska, 2013). De hecho, en el presente estudio se encontró que ambas subescalas para los puntos de corte propuestos para el diagnóstico diferencial detectan un porcentaje importante de pacientes como "falsos positivos".

La idea vigente en los manuales de diagnóstico supone la visión de que ambos son trastornos diferentes y limitados en el tiempo; sin embargo, las críticas al sistema categorial se encuentran desde el mismo DSM-IV-R (American Psychiatric Association, 2002) en el que se indica por ejemplo, que no debe asumirse que las categorías clínicas sean entidades discretas con límites absolutos, lo que también se ha considerado para el DSM-V (García et al., 2011). Así mismo, otros autores ponen en duda la hipótesis de que los síndromes representan diferentes etiologías, sobre todo en lo relativo a los trastornos adaptativos de tipo ansioso o depresivo (Grau, Martín, \& Portero 1993; Fountoulakis, 2010; Batterham, Christensen, \& Calear, 2013).

De acuerdo con el DSM-IV-TR (American Psychiatric Association, 2002) solo un pequeño número de síntomas relacionados con la ansiedad son capaces de distinguir entre personas ansiosas y personas depresivas. Esta limitación no ha sido superada en el DSM-V, como ya constatan algunos estudios (e.g., Angst, Hengartner, Ajdacic-Gross, \& Rössler, 2014). Por otra parte, Fried, Nesse, Zivin, Guille, \& Sen (2013) cuestionan la idea de que los síntomas que se proponen para el diagnóstico de la depresión sean indicadores de un trastorno único subyacente, al constatar en un estudio longitudinal, que su asociación con los factores de riesgo es independiente del diagnóstico.

Para complicar más las cosas, la comorbilidad ha sido relacionada con variables como la cronicidad y la gravedad de los trastornos, los resultados del tratamiento y las recaídas, la búsqueda de tratamiento, el riesgo de suicidio y el funcionamiento general de los pacientes (Bair et al., 2013)

También debe recordarse la relevancia clínica y social que tiene el trastorno mixto de ansiedad y depresión, como recientemente ha mostrado el propio Goldberg en un estudio epidemiológico (Das-Munshi, Goldberg, Bebbington, Bhugra, \& Bhugra, 2008), lo que orienta a que se sea sumamente cuidadoso en el diagnóstico diferencial.

En conclusión, este estudio avala la posibilidad de utilizar la EAD-G tanto para el cribaje como para la identificación de los trastornos ansiosos y depresivos, aunque esto último con reservas debido a los problemas teóricos y metodológicos involucrados en la diferenciación de estos trastornos. Deberá continuarse la validación con muestras representativas de la población cubana, obteniendo otros criterios de validez externa como por ejemplo, su correlación con otros instrumentos para el diagnóstico de la ansiedad y la depresión. 


\section{Referencias}

American Psychiatric Association (2002). Manual Diagnóstico y Estadístico de los Trastornos Mentales. Cuarta edición revisada. Mason: Barcelona.

Angst, J., Hengartner, M.P., Ajdacic-Gross, V., \& Rössler, G. (2014). iEl criterio de duración de dos semanas es el óptimo para la depresión mayor? Actas Españolas de Psiquiatría, 42(1), 18-27.

Arrol, B., Khin, N., \& Kerse, N. (2003). Screening for depression in primary care with two verbally asked questions: cross sectional study. British Medical Journal, 327, 1144-1146. http://dx.doi.org/10.1136/ bmj.327.7424.1144

Asociación Psiquiátrica de América Latina - APAL(2003). Guía Latinoamericana de Diagnóstico Psiquiátrico. Organización Mundial de la Salud.

Australian Longitudinal Study on Women's HealthALSWH (2011). Performance of the Goldberg Anxiety and Depression Scale in older women. Recuperado el 1 de marzo de 2013, de http:// ageing.oxfordjournals.org/content/37/4/464. full.pdf + html?sid=a09ac381-5535-4101-9acee68f0e126f6d

Baca, E., Saiz, J., Agüera, L., Caballero, L., FernándezLiria, A., Ramos, J.,...Porras, A. (1999). Validación de la versión española del PRIME-MD: un procedimiento para el diagnóstico de trastornos mentales en atención primaria. Actas Españolas de Psiquiatría, 27(6), 375-383.

Bair, M. J., Poleshuck, E. L., Wu, J., Krebs, E.K., Damush, T., Tu, W., \& Kroenke, K. (2013). Anxiety but Not Social Stressors Predict 12-Month Depression and Pain Severity. Clinical Journal of Pain, 29(2), 95-101. http://dx.doi.org/10.1097/AJP.0b013e3182652ee9.

Ballenger, J.C., Davidson, J.R.T., Lecrubier, Y., Nutt, D.J., Kirmayer, L.J., Lepine, J.P.,...Ono, Y. (2001). Consensus statement on transcultural issues in depression and anxiety from the International Consensus Group on Depression and Anxiety. Journal of Clinical Psychiatry, 62(13), 47-55.

Batterham, J.P., Christensen, H., \& Calear, A.L. (2013) Anxiety symptoms as precursors of major depression and suicidal ideation. Journal of Depression and anxiety, 30(10), 908-916. http://dx.doi.org/10.1002/ da. 22066
Botto, A., Acuña, J., \& Jiménez, J.A. (2014). La depresión como un diagnóstico complejo. Implicancias para el desarrollo de recomendaciones clínicas. Revista Médica de Chile, 142 (10), 1297-1305. http:// dx.doi.org/10.4067/S0034-98872014001000010.

Tizón, J.L., Buitrago, F., Ciurana, R., Chocrón, L., Fernández, C., García, J.,... Redondo, M.J. (1999). Prevención de los trastornos de la salud mental desde la atención primaria de salud. Atención Primaria, 24(1), 184-190.

Bunevicius, A., Peceliuniene, J., Mickuviene, N., Valius, L., \& Bunevicius, R. (2007). Screening for depression and anxiety disorders in primary care patients. Depression E Anxiety, 24(7), 455-60.

Cameron, I.M., Crawford, J.R., \& Lawton, K. (2008). Psychometric comparison of PHQ-9 and HADS for measuring depression severity in primary care. British Journal of General Practice, 58(546), 32-36.

Cano, A., Martín, J., Wood, C.M., Dongil, E., \& Latorre, J.M. (2012). La depresión en atención primaria: prevalencia, diagnóstico y tratamiento. Papeles del Psicólogo, 33(1), 2-11.

Cepoiu, M., McCusker, J., Cole, M.G., Sewitch, M., Belzile, \& E., Ciampi, A. (2008). Recognition of depression by non-psychiatric physicians: a systematic literature review and meta-analysis. Journal of General Internal Medicine, 23(1), 25-36.

Fernández, M.C., Buitrago, F., Ciurana, R., Chocrón, L., García, J., Montón, C., \& Tizón J.L. (2007). Programa de Prevención en Salud Mental en Atención Primaria. Atención Primaria, 39, 88-108.

Fernández, A., Pinto-Meza, A., Bellón, J.A., RouraPoch, P., Haro, J.M., Autonell, J.,... Serrano-Blanco, A. (2010) Is major depression adequately diagnosed and treated by general practititoners? Results from an epidemiological study. General Hospital Psychiatry, 32(2), 201-209.

Fors, M.M. (2011). Guía de práctica clínica para el manejo de episodios depresivos y trastornos depresivos recurrentes en pacientes entre 18 y 64 años. (Tesis Doctoral). La Habana: manuscrito no publicado.

Franco, N., \& Vivo, J.M. (2007). Análisis de curvas ROC. Principios básicos y aplicaciones. Madrid: La Muralla.

Fried, E., Nesse, R.M., Zivin, K., Guille, C., \& Sen, S. (2013). Depression is more than the sum score 
of its parts: individual DSM. Psychological Medicine, 1-10, Available on CJO 2013 http://dx.doi. org/10.1017/S0033291713002900.

García, G., Saldívar, A.H., LLanes, A., \& Sánchez, I.G. (2011). El DSM-V. Luces y sombras de un manual no publicado. Retos y expectativas para el futuro. Salud Mental, 34(4), 367-378.

Gilbody, S., House, A., \& Sheldon, T. (2009). Screening and case finding instruments for depression. Cochrane Database of Systematic Reviews. The Cochrane Library, Issue 11, Art. No. CD002792. http://dx.doi.org/10.1002/14651858.CD002792. pub4.

Goldberg D, Bridges K, Duncan-Jones P, Grayson D. (1987). Dimensions of neurosis seen in primary care settings. Psychological Medicine, 17, 461-470.

Goldberg, D., Bridges, K., Duncan-Jones, P., \& Grayson, D. (1988). Detecting anxiety and depression in general medical settings. British Medical Journal, (6653), 897-899.

Grau, J.A., Martín, M. \& Portero, D. (1993). Estrés, ansiedad, personalidad: resultado de las investigaciones cubanas. Revista Interamericana de Psicología, 27(1), 37-58.

Gruszczyńska, E. (2013). State affect and emotionfocused coping: examining correlated change and causality. Anxiety, stress and coping 26(1), 103-119. http://dx.doi.org/10.1080/10615806.2011.631526.

Kirmayer, L.J. (2001). Cultural variations in the clinical presentation of depression and anxiety: implications for diagnosis and treatment. Journal of Clinical Psychiatry, 62(13), 22-68.

Kohn, R., Levav, I., Caldas de Almeida, J.M., Vicente, B., Andrade, L., Caraveo-Anduaga, J.J.....Saraceno, S. (2005). Los trastornos mentales en América Latina y el Caribe: asunto prioritario para la salud pública. Revista Panamericana de Salud Pública, 18(4/5), 229-40.

Fountoulakis, K. (2010). The emerging modern face of mood disorders: a didactic editorial with a detailed presentation of data and definitions. Annals of General Psychiatry, 9, 14, 1-22. http://dx.doi. org/10.1186/1744-859X-9-14.

Latorre, J.M., Navarro, B., Parra, M., Salguero, J.M., Mae Wood, C., \& Cano, A. (2012). Evaluación e intervención de los problemas de Ansiedad y Depresión en Atención Primaria: Un problema sin resolver. Revista Clínica de Medicina Familiar, 5(1), 37-45.

Lobo, A., Montón, A., Campos, R., García-Campayo, J., Pérez, Echevarría, M.J. (1993). Detección de morbilidad psíquica en la práctica médica: el nuevo instrumento E.A.D.G. Zaragoza: Luzán;

López, M., Gabarrón, R., \& Ruiz, A. (febrero-marzo de 2011). Depresión en atención primaria: una aproximación a los trabajos realizados en España. Trabajo presentado en el XII Congreso Virtual de Psiquiatría Interpsiquis 2011. Resumen recuperado el 22 de diciembre de 2012, de http://www. psiquiatria.com/revistas/index.php/psiquiatriacom/article/download/1211/1121

Mackinnon, A., Christensen, H., Form, A. (1994). A latent trait analysis of an inventory designed to detect symptoms of anxiety and depression using an elderly community sample. Psychological Medicine, 24, 977-86.

Magnavita, N. (2007). Anxiety and depression at work. the A/D Goldberg Questionnaire. Giornale Italiano di Medicina del Lavoro ed Ergonomia, 29(3), 670-671.

Manifesto por uma psicopatologia clínica não estatística. (2013). Revista Latinoamericana de Psicopatología Fundamental, 16(3), 361-372. http://dx.doi. org/10.1590/S1415-47142013000300001.

Martínez-Bernardos, R., Baylín-Larios, A., Ortiz-Jiménez, M.F. (1999). ¿Podemos detectar trastornos mentales en atención primaria? Utilidad y concordancia de dos instrumentos diagnósticos. Atención Primaria, 23, 285-288.

Martínez-García, J.A., \& Martínez-Caro, L. (2009). La validez discriminante como criterio de evaluación de escalas: iteoría o estadística? Universitas Psychologica, 8(1), 27-36.

McGorry, P., \& Van Os, J. (2013). Reconsiderando el diagnóstico en Psiquiatría. Lancet, 381, 343-345.

Mitchell, A.J., Vaze, A., \& Rao S. (2009) Clinical diagnosis of depression in primary care: a metaanalysis. Lancet, 374, 609-619.

Montón, C., Pérez-Echevarría, M.J., \& Campos, R (1993). Escalas de ansiedad y depresión de Goldberg: una guía de entrevista eficaz para la detección del malestar psíquico. Atención Primaria, 12, 345-349. 
Das-Munshi, J.D., Goldberg, D., Bebbington, P.E., Bhugra, D.K., \& Brugha, T.S., Dewey, M.E....Prince, M. (2008). Public health significance of mixed anxiety and depression: beyond current classification. British Journal of Psychiatry, 192(3), 171-177. http://dx.doi.org/10.1192/bjp.bp.107.036707.

Muñiz, J. (1997). Introducción a la Teoría de Respuesta a los Items. Madrid: Pirámide.

Rasch, G. (1960). Probabilistic models for some intelligence and attainment tests. Copenhagen: Danish Institute of Educational Research.

Sandín, B., Chorot, P., Lostao, L., Joiner, T.E., Santed, M.A., \& Valiente, R. (1999). Escalas PANAS de afecto positivo y negativo: validación factorial y convergencia transcultural. Psicothema, 11(1), $37-51$.

van Beljouw, I.M., Verhaak, P.M., Cuijpers, P., van Marwijk, H.W., \& Penninx, B.W. (2010). The course of untreated anxiety and depression and determinants of poor one-year outcome: a one-year cohort study. Biomed Central Psychiatry, 10-86. http:// dx.doi.org/.10.1186/1471-244X-10-86.

Watson, D., Clark, L. A., \& Tellegen, A. (1988). Development and validation of brief measures of Positive and Negative Affect: The PANAS Scales. Journal of Personality and Social Psychology, 54, 1063-1070. 
